\title{
Synthesis and insecticidal activity of 1,2,4-triazole derivatives
}

\author{
Bing Chai ${ }^{\mathrm{a}}$, Xuhong Qian ${ }^{\mathrm{b}}$, Song Cao ${ }^{\mathrm{a}}$, Haidong Liu ${ }^{\mathrm{a}}$, Gonghua Song ${ }^{\mathrm{a}}$ \\ ${ }^{a}$ Institute of Pesticides and Pharmaceuticals, East China University of Science and \\ Technology,P.O. Box 544, 130 Meilong Road, Shanghai 200237, PR China \\ ${ }^{\mathrm{b}}$ State Key Laboratory of Fine Chemicals, Dalian University of Technology, Dalian 116012, PR \\ China \\ E-mail:xhqian@dlut.edu.cn \\ (received 21 Feb 03; accepted 10 May 03; published on the web 14 May 03)
}

\begin{abstract}
Eight novel compounds were synthesized and their insecticidal activities were tested. The compounds of 3d, 3e and 3g showed insecticidal activity against Aphis rumicis Linnaeus. All the title compounds were characterized on the basis of IR, ${ }^{1} \mathrm{H}$ NMR and HRMS.
\end{abstract}

Keywords: Synthesis, insecticidal activity, 1,2,4-triazole

\section{Introduction}

The azole moiety is an important and frequent insecticidal, agrochemical structural feature of many biologically active compounds such as cytochrome P450 enzyme inhibitors ${ }^{1}$ and peptide analog inhibitors ${ }^{2}$. Recently, much attention has been focused on 1H-1,2,4-triazole derivatives for their broad-spectrum activities, such as fungicidal, herbicidal, anticonvulsant and plant growth regulatory activities $^{3-5}$. Further, the disubstituted 1,2,4-triazole derivatives were also reported to show antifungal, insecticidal, herbicidal and anti-inflammatory properties which were similar to $1 \mathrm{H}-1,2,4$-triazole derivatives ${ }^{6-8}$. Promoted by the above observations that the combination of two or more heterocyclic and non-heterocyclic systems enhances the biological profile many-fold than its parent nuclei, we considered to synthesize some compounds bearing 1H-1,2,4-triazole in a molecular framework. 3(2H)-Pyridazinones and their derivatives have been reported having fungicidal, insecticidal and pharmacological activities ${ }^{9-11}$. Some of them showed similar activity as $\mathrm{JH}^{12-13}$, such as $\mathrm{NC}-170$ and $\mathrm{NC}-184$. (Scheme 1) Taking these structural features into consideration, it was thought worthwhile to synthesize the novel compounds that combining the 1,2,4-triazole with 2-t-butyl-4,5-dichloro-pyridazinone by sulfur heteroatom. 
<smiles>N#C[In]c1cnn(-c2ccc(Cl)c(Cl)c2)c(=O)c1Cl</smiles><smiles>CC(C)(C)Cn1ncc(OCc2ccc(I)nc2)c(Cl)c1=O</smiles>

\section{Scheme 1}

\section{Results and Discussion}

The synthetic route of the title compounds was seen in Scheme 2.

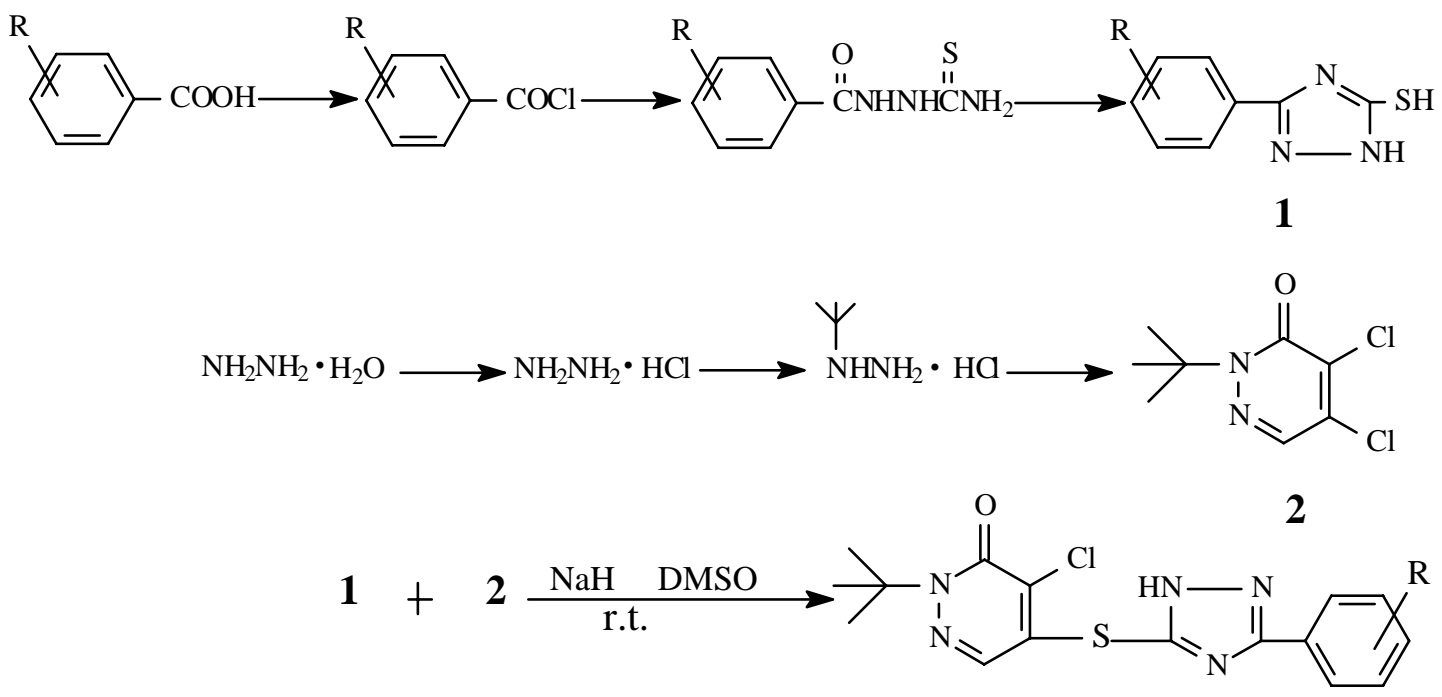

3

3a $\mathrm{R}=\mathrm{H} ; 3 \mathrm{~b} \mathrm{R}=2-\mathrm{OCH}_{3} ; 3 \mathrm{c} \mathrm{R}=4-\mathrm{NO}_{2} ; 3 \mathrm{~d} \mathrm{R}=3-\mathrm{NO}_{2} ; 3 e \mathrm{R}=4-\mathrm{OCH}_{3}$;

3f $\mathrm{R}=3,5-2 \mathrm{CH}_{3} ; 3 \mathrm{~g} \mathrm{R}=4-\mathrm{F}$; 3h $\mathrm{R}=3-\mathrm{F}$

\section{Scheme 2}

The intermediates, $\mathbf{1}$ and $\mathbf{2}$, were synthesized according to the literature ${ }^{14,15}$ and got the accept yield. The synthetic conditions of the title compounds were investigated, such as temperature, time and base. The temperature had great effect on the yield of the title compounds. The low temperature will prolong the reactive time, otherwise, the by-product will become more when the temperature is beyond $40^{\circ} \mathrm{C}$. We hold the room temperature about $30^{\circ} \mathrm{C}$ and got the satisfied yield. The effect of strong base was better than that of weak base clearly.

General Preparation of the title compounds 3a-h: 1 (2 mmol) and $\mathrm{NaH}(2 \mathrm{mmol})$ were solved in DMSO $(10 \mathrm{~mL})$. The mixture were stirred for 1 hour in the flask $(50 \mathrm{~mL})$ by magnetic stirrer at 
the room temperature. Then, $2(2 \mathrm{mmol})$ was added into the mixture and continued stirring for about 10 hours. The reactive process was monitored by TLC until the starting material nearly disappeared. The reactive mixture was poured into water $(20 \mathrm{~mL})$, extracted by chloroform (30 $\mathrm{mL}$ ), drying over $\mathrm{MgSO}_{4}$, the solvent was filtered and evaporated under the reduced pressure, the residue was purified by flash chromatography (petroleum ether/ethyl acetate $=2: 1$ ) to afford the title compound then crystallized from methanol.

Judging from the structure of the intermediate 2, the product should contain another isomer which substituted the 4-Cl atom. But, the TLC results showed that there is only one new compound during the whole reactive process, and we only get one substituted compound through the flash chromatography. The reason might be that the carbonyl group of the pyridazinone moiety make the reactivity of 5-C atom is higher than that of 4-C atom. So, we don't find the compound substituted the 4-Cl atom.

All the title compounds' bioactivity was screened by the method of leaf-dip. A stock solution of title compounds (1000ppm) in DMSO was used for preparing various concentrations for bioactivity screening. The compounds of $\mathbf{3 d}$, $\mathbf{3 e}$ and $\mathbf{3 g}$ showed insecticidal activity against Aphis rumicis Linnaeus. They have the insecticidal rate $45 \%, 38 \%$ and $30 \%$ at the concentration of 500ppm. The insecticidal activity decreased clearly when the concentration was decreased.

\section{Experimental Section}

General Procedures. Infrared spectra were taken on a Nicolet FT-IR-20SX spectrometer using $\mathrm{KBr}$ disks; HRMS spectra on a Micromass GCT instrument; and ${ }^{1} \mathrm{H}$ NMR spectra on a Brucker WP500SY(500 MHz) spectrometer with DMSO- $\mathrm{d}_{6}$ or $\mathrm{CDCl}_{3}$ as solvent and TMS as internal standard. Melting points were measured by a digital melting point apparatus made in Shanghai and were uncorrected. All reactions were followed by TLC.

Materials. Unless otherwise stated, these were commercial samples. All organic solvents were of analytical quality and used as purchased. Solvent mixtures are defined by volume ratios (v/v).

2-t-Butyl-4-chloro-5[(3-phenyl-1H-1,2,4-triazol-5yl)thio]pyridazin-3(2H)-one (3a). mp 126$7^{\circ} \mathrm{C}$ yield 60\% IR (KBr cm ${ }^{-1}$ ): $3400(\mathrm{NH}) 1690(\mathrm{CN})$ 1590(CC) 1650(CO) ${ }^{1} \mathrm{H}$ NMR $\left(\mathrm{CDCl}_{3}\right): \delta$ 1.62 (s, 9H, t-butyl) 7.51-7.53 (d, 3H, J=1.1 Hz, Ar-H) 7.5 (s, 1H, Py-H) 8.06-8.07 (dd, 2H, $J=1.6,2.3 \mathrm{~Hz}$, Ar-H) HRMS: Calc. for: $\mathrm{C}_{16} \mathrm{H}_{16} \mathrm{Cl}^{35} \mathrm{~N}_{5} \mathrm{OS} 361.0764$ Found: 361.0765 Calc. for: $\mathrm{C}_{16} \mathrm{H}_{16} \mathrm{Cl}^{37} \mathrm{~N}_{5} \mathrm{OS} \quad 363.0735$ Found: $363.0746 \mathrm{MS}: 361\left(\mathrm{M}^{+}, 5\right), 326(35), 270(100), 242(43)$, 306(19), 177(10), 104(18).

2-t-Butyl-4-chloro-5[(3-(3-methoxyphenyl)-1H-1,2,4-triazol-5yl)thio]pyridazin-3(2H)-one (3b). mp 237-8 ${ }^{\circ} \mathrm{C}$ yield 58\% IR ( $\left.\mathrm{KBr} \mathrm{cm}^{-1}\right): 3400(\mathrm{NH})$ 1690(CN) 1590(CC) 1650(CO) ${ }^{1} \mathrm{H}$ NMR (CDCl $): \delta 1.62$ (s, 9H, t-butyl) 4.11 (s, 3H, $\left.-\mathrm{OCH}_{3}\right) 7.43$ (s, 1H, Py-H) 7.1-8.4 (m, 4H, Ar-H) HRMS: Calc. for: $\mathrm{C}_{17} \mathrm{H}_{18} \mathrm{Cl}^{35} \mathrm{~N}_{5} \mathrm{O}_{2} \mathrm{~S} 391.0870$ Found: 391.0903 Calc. for: 
$\mathrm{C}_{17} \mathrm{H}_{18} \mathrm{Cl}^{37} \mathrm{~N}_{5} \mathrm{O}_{2} \mathrm{~S} \quad 393.0840$ Found: $393.0849 \mathrm{MS}: 391\left(\mathrm{M}^{+}, 6\right), 356(49), 300(100), 272(63)$, 336(14), 134(18).

2-t-Butyl-4-chloro-5[(3-(4-nitrophenyl)-1H-1,2,4-triazol-5yl)thio]pyridazin-3(2H)-one (3c). mp 264-5 ${ }^{\circ} \mathrm{C}$ yield 63\% IR (KBr cm $\left.{ }^{-1}\right): 3400(\mathrm{NH}) 1690(\mathrm{C}=\mathrm{N}) 1590(\mathrm{C}=\mathrm{C}) 1650(\mathrm{C}=\mathrm{O}){ }^{1} \mathrm{H} \mathrm{NMR}$ $\left(\mathrm{CDCl}_{3}\right): \delta 1.55$ (s, 9H, t-butyl) 7.53 (s, 1H, Py-H) 8.27-8.28 (d, 2H, J=8.8 Hz, Ar-H) 8.42-8.43 (d, $2 \mathrm{H}, \mathrm{J}=8.6 \mathrm{~Hz}, \mathrm{Ar}-\mathrm{H}$ ) HRMS: Calc. for: $\mathrm{C}_{16} \mathrm{H}_{15} \mathrm{Cl}^{35} \mathrm{~N}_{6} \mathrm{O}_{3} \mathrm{~S} 406.0613$ Found: 406.0615 MS: 406( $\left.\mathrm{M}^{+}, 2\right)$, 371(9), 351 (23), 315(100), 287(18), 57(5).

2-t-Butyl-4-chloro-5[(3-(3-nitrophenyl)-1H-1,2,4-triazol-5yl)thio]pyridazin-3(2H)-one (3d). mp 161-2 ${ }^{\circ} \mathrm{C}$ yield 54\% IR (KBr cm $\left.{ }^{-1}\right): 3400(\mathrm{NH}) 1690(\mathrm{C}=\mathrm{N}) 1590(\mathrm{C}=\mathrm{C}) 1650(\mathrm{C}=\mathrm{O}){ }^{1} \mathrm{H}$ NMR $\left(\mathrm{CDCl}_{3}\right): \delta 1.56$ (s, 9H, t-butyl) 7.52 (s, 1H, Py-H) 7.87-7.90 (m, 1H, Ar-H) 8.39-8.40 (d, 1H, J=7.7 Hz, Ar-H) 8.44-8.45 (d, 1H, J=7.8 Hz, Ar-H) 8.86 (s, 1H, Ar-H) HRMS: Calc. for: $\mathrm{C}_{16} \mathrm{H}_{15} \mathrm{Cl}^{35} \mathrm{~N}_{6} \mathrm{O}_{3} \mathrm{~S}$ 406.0615 Found: 406.0639 MS: 406( $\left.\mathrm{M}^{+}, 2\right), 371(8), 351$ (23), 315(100), 287(16), 149(11).

\section{2-t-Butyl-4-chloro-5[(3-(4-methoxyphenyl)-1H-1,2,4-triazol-5yl)thio]pyridazin-3(2H)-one}

(3e). mp 210-1 ${ }^{\circ} \mathrm{C}$ yield 67\% IR (KBr cm $\left.{ }^{-1}\right)$ : $3400(\mathrm{NH}) 1690(\mathrm{C}=\mathrm{N}) 1590(\mathrm{C}=\mathrm{C}) 1650(\mathrm{C}=\mathrm{O}){ }^{1} \mathrm{H}$ NMR (CDCl $)_{3}$ ): $\delta 1.54$ (s, 9H, t-butyl) 3.80 (s, 3H, - $\left.\mathrm{OCH}_{3}\right) 7.27$ (s, 1H, Py-H) 7.00-7.01 (d, 2H, $J=8.8 \mathrm{~Hz}, \mathrm{Ar}-\mathrm{H}) 7.93$ (d, 2H, $=8.8 \mathrm{~Hz}, \mathrm{Ar}-\mathrm{H})$ HRMS: Calc. for: $\mathrm{C}_{17} \mathrm{H}_{18} \mathrm{Cl}^{35} \mathrm{~N}_{5} \mathrm{O}_{2} \mathrm{~S} 391.0870$ Found: 391.0890 Calc. for: $\mathrm{C}_{17} \mathrm{H}_{18} \mathrm{Cl}^{37} \mathrm{~N}_{5} \mathrm{O}_{2} \mathrm{~S} 393.0840$ Found: 393.0892 MS: 391( $\left.\mathrm{M}^{+}, 22\right)$, 356(46), 300(100), 272(57), 335(25), 134(41).

2-t-Butyl-4-chloro-5[(3-(3,5-dimethylphenyl)-1H-1,2,4-triazol-5yl)thio]pyridazin-3(2H)-one (3f). mp 195- $7^{\circ} \mathrm{C}$ yield 65\% IR $\left(\mathrm{KBr} \mathrm{cm}^{-1}\right)$ : $3400(\mathrm{NH}) 1690(\mathrm{C}=\mathrm{N}) 1590(\mathrm{C}=\mathrm{C}) 1650(\mathrm{C}=\mathrm{O}){ }^{1} \mathrm{H}$ NMR $\left(\mathrm{CDCl}_{3}\right.$ ): $\delta 1.51$ (s, 9H, t-butyl) 2.31 (s, 6H, 2Ar-CH $) 7.13$ (s, 1H, Py-H) 7.42 (s, 1H, ArH) 7.62 (s, 2H, Ar-H) HRMS: Calc. for: $\mathrm{C}_{18} \mathrm{H}_{20} \mathrm{Cl}^{35} \mathrm{~N}_{5} \mathrm{OS} 389.1077$ Found: 389.1079 MS: 389 (M+ 8), 354(50), 298(100), 270 (51), 334 (13), 205 (7)

2-t-Butyl-4-chloro-5[(3-(4-fluorophenyl)-1H-1,2,4-triazol-5yl)thio]pyridazin-3(2H)-one (3g). mp 164- $6^{\circ} \mathrm{C}$ yield 72\% IR (KBr cm$\left.{ }^{-1}\right)$ : $3400(\mathrm{NH}) 1690(\mathrm{C}=\mathrm{N}) 1590(\mathrm{C}=\mathrm{C}) 1650(\mathrm{C}=\mathrm{O}){ }^{1} \mathrm{H}$ NMR $\left(\mathrm{CDCl}_{3}\right): \delta 1.55$ (s, 9H, t-butyl) 7.49 (s, 1H, Py-H) 7.32-7.46 (m, 2H, Ar-H) 8.04-8.09 (m, 2H, Ar-H) HRMS: Calc. for: $\mathrm{C}_{16} \mathrm{H}_{15} \mathrm{Cl}^{35} \mathrm{FN}_{5} \mathrm{OS} 379.0670$ Found: $379.0670 \mathrm{MS}: 379\left(\mathrm{M}^{+}, 8\right), 344$ (42), 288 (100), 260 (61), 324 (28), 122 (19).

2-t-butyl-4-chloro-5[(3-(3-fluorophenyl)-1H-1,2,4-triazol-5yl)thio]pyridazin-3(2H)-one (3h). mp 170-1 ${ }^{\circ} \mathrm{C}$ yield 69\% IR (KBr cm $\left.{ }^{-1}\right): 3400(\mathrm{NH})$ 1690(C=N) 1590(C=C) 1650(C=O) ${ }^{1} \mathrm{H}$ NMR $\left(\mathrm{CDCl}_{3}\right): \delta 1.56$ (s, 9H, t-butyl) 7.52 (s, 1H, Py-H) 7.32-7.50 (m, 2H, Ar-H) 8.00-8.02 (m, 2H, Ar-H) HRMS: Calc. for: $\mathrm{C}_{16} \mathrm{H}_{15} \mathrm{Cl}^{35} \mathrm{FN}_{5} \mathrm{OS} 379.0670$ Found: $379.0672 \mathrm{MS}: 379\left(\mathrm{M}^{+}, 14\right), 344$ (27), 324 (48), 288 (100), 260 (90), 122 (29).

\section{Acknowledgements}


We express our gratitude to the National Nature Science Foundation of China and Shanghai Foundation of Science and Technology for the partial financial support.

\section{References}

1. Vanden Bossche, H. J.; Steroid Biochem. Molec. Biol. 1992, 42, 45.

2. Meek, T. D.; J. Enzyme Inhib. 1992, 6, 65.

3. Toyabe, K.; Nezu, M.; Shimazu, H.; Jpn Kokai Tokkyo Koho Jp, 0641086; Chem. Abstr, 1989,121, 9409q.

4. Shaber, S. J.; Flyn, K. E.; Fujimoto, T. T.; Eur Pat Ep, 529, 973 Chem. Abstr., 1993, 119, $72612 \mathrm{z}$.

5. Stankovsky, S.; Jedlovska, E.; Spirkova, K.; Collcct Czech Chem. Commun. 1993, 58, 2211.

6. Talawar, M. B.; Laddi, U. V.; Somannavar, Y. S.; Benner, R. S.; Bennur, S. C. Indian J. Heterocycl. Chem. 1995, 4, 297.

7. Zhang, Z. Y.; Yan, H.; Acta Chimica Sinica 1987, 45, 403.

8. Talawar, M. B.; Bennur, S. C.; Kankanwadi, S. K.; Patil P. A., Indian J. Pharm Sci. 1995, 57, 194.

9. Egan, A. R.; Eur. Pat. Appl. EP 478,195 Chem. Abstr, 1992, 118, $131213 d$.

10. Umehara, T.; Jpn. Kohai Tokkyo Koho JP 03,220,177 Chem. Abstr, 1992, 118, $21926 f$.

11. Tanikawa, K.; PCT Int. Appl. WO 91 16,314 Chem. Abstr, 1992, 118, 106307j.

12. Schnal, F.; The Juvenile Hormones, Gilbert L. I., Plenum, New York, 1976, p301.

13. Biwers, W. S.; Sugiyama, T.; WO 9006678, 1990m Chem. Abstr, 1988, 114, 37808.

14. Shi, H. J.; Wang, Z. Y.; Shi, H. X.; YOUJI HUAXUE 1996, 16, 242

15. Dury, K.; Angew. Chem. Int. Ed. 1965, 4, 292. 